\title{
New criteria for the existence of non-trivial fixed points in cones
}

\author{
Alberto Cabada ${ }^{1}$, José Ángel Cid ${ }^{2 *}$ and Gennaro Infante ${ }^{3}$
}

${ }^{\text {"Correspondence: }}$ angelcid@uvigo.es

2Departamento de Matemáticas, Universidade de Vigo, Pabellón 3 (Edifico Físicas), Campus de Ourense, Ourense, 32004, Spain Full list of author information is available at the end of the article

\begin{abstract}
We give new criteria for the existence of nontrivial fixed points on cones assuming some monotonicity of the operator on a suitable conical shell. Moreover, we give an application to the existence of multiple solutions for a nonlocal boundary value problem that models the displacement of a beam subject to some feedback controllers.
\end{abstract}

MSC: 47H10; 34B10; 34B18

Keywords: Krasnosel'skiĭ fixed point theorem; positive solutions; conical shells; multiplicity

\section{Introduction and preliminaries}

The classical cone compression-expansion fixed point theorem of Krasnosel'skii (see Theorem 1.2 below) and the monotone iterative technique (see Theorem 1.1 below) are among the most popular and fruitful tools to deal with the existence of solutions for nonlinear problems. Following earlier ideas of Persson [1] valid in the finite-dimensional setting, both methods were combined in [2] to obtain the existence of a fixed point, assuming the operator $T$ to be monotone non-decreasing with some conditions on the set of supersolutions. This result was improved in [3] by relaxing the monotonicity condition. More recently, in [4] the authors were able to present a refinement of the results of [2, 3], by allowing a comparison between a point and a boundary, instead of on two boundaries as in Krasnosel'skiî's theorem. This approach, which required a monotonicity assumption on the operator on a conical shell, has proved to be well suited to establish multiplicity results. Our aim in this paper is to pursue this line of research by obtaining new fixed point theorems, valid not only for non-decreasing (Section 3) but also for non-increasing operators (Section 2). We point out that this type of theorems can be combined in the applications to obtain the existence of multiple non-trivial solutions. This fact is illustrated in Section 4, where the existence of multiple positive solutions for a nonlocal boundary value problem modeling the displacement of a beam is discussed.

We now recall some definitions that will be useful in the sequel. A subset $K$ of a real Banach space $N$ is a cone if and only if it is closed, $K+K \subset K, \lambda K \subset K$ for all $\lambda \geq 0$ and $K \cap(-K)=\{0\}$. A cone $K$ defines the partial ordering in $N$ given by $x \leq y$ if and only if $y-x \in K$. The notation $x<y$ means $x \leq y$ and $y \neq x$. The cone $K$ is called normal with a normal constant $c \geq 1$ if and only if $\|x\| \leq c\|y\|$ for all $x, y \in N$ with $0 \leq x \leq y$. Whenever $\operatorname{int}(K) \neq \emptyset$, the symbol $x \ll y$ means $y-x \in \operatorname{int}(K)$ and the cone is said to be solid. $\partial K$ denotes the boundary of $K$ and $d(x, \partial K)$ is the distance of $x$ to the boundary of $K$.

(c) 2013 Cabada et al.; licensee Springer. This is an Open Access article distributed under the terms of the Creative Commons Attribution License (http://creativecommons.org/licenses/by/2.0), which permits unrestricted use, distribution, and reproduction in any medium, provided the original work is properly cited. 
If $T: K \rightarrow K$ satisfies the conditions

$T x \nless x \quad$ for all $x \in K$ with $\|x\|=R$

and

$T x \ngtr x \quad$ for all $x \in K$ with $\|x\|=\bar{R}$,

then it is called a cone compression when $0<R<\bar{R}$ and a cone expansion when $0<\bar{R}<R$.

The closed ball of center $x_{0} \in N$ and radius $r>0$ is denoted by

$$
B\left[x_{0}, r\right]=\left\{x \in N:\left\|x-x_{0}\right\| \leq r\right\},
$$

and for $x, y \in N$, with $x \leq y$, we define the interval

$$
[x, y]=\{z \in N: x \leq z \leq y\} .
$$

Now we recall the two classical fixed point results mentioned above. The first one is known as the monotone iterative method (see, for example, [5, Theorem 7.A] or [6]).

Theorem 1.1 Let $N$ be a real Banach space with a normal order cone K. Suppose that there exist $\alpha \leq \beta$ such that $T:[\alpha, \beta] \subset N \rightarrow N$ is a completely continuous monotone nondecreasing operator with $\alpha \leq T \alpha$ and $T \beta \leq \beta$. Then $T$ has a fixed point and the iterative sequence $\alpha_{n+1}=T \alpha_{n}$, with $\alpha_{0}=\alpha$, converges to the greatest fixed point of $T$ in $[\alpha, \beta]$, and the sequence $\beta_{n+1}=T \beta_{n}$, with $\beta_{0}=\beta$, converges to the smallest fixed point of $T$ in $[\alpha, \beta]$.

The second one, which is widely used for the search of positive fixed points, is due to Krasnosel'skii (see [5, Theorem 13.D]).

Theorem 1.2 Let $N$ be a real Banach space with order cone K. Suppose that the operator $T: K \rightarrow K$ is completely continuous and either a cone compression or expansion. Then $T$ has a fixed point $x$ on $K$ and

$$
\min \{R, \bar{R}\}<\|x\|<\max \{R, \bar{R}\} .
$$

\section{Non-increasing operators}

Firstly, we present a result under the assumption of the existence of a lower solution in a solid cone.

Theorem 2.1 Let $N$ be a real Banach space, $K$ be a solid cone and $T: K \rightarrow K$ be a completely continuous operator. Assume that

(1) there exist $\alpha_{1} \in K$, with $\alpha_{1} \leq T \alpha_{1}$, and $R_{1}>0$ such that $B\left[\alpha_{1}, R_{1}\right] \subset K$;

(2) the map $T$ is monotone non-increasing in the set

$$
K_{2}=\left\{x \in K: R_{1} \leq\|x\| \leq\left\|\alpha_{1}\right\|\right\}
$$

(3) there exists $r_{1}>0$, with $r_{1} \neq R_{1}$, such that $T x \nsupseteq x$ for all $x \in K$ with $\|x\|=r_{1}$. 
Then the map $T$ has at least one non-zero fixed point $x_{1} \in K \backslash\left\{x \in K ;\|x\|=r_{1}\right\}$ such that

$$
\min \left\{r_{1}, R_{1}\right\} \leq\left\|x_{1}\right\| \leq \max \left\{r_{1}, R_{1}\right\}
$$

Proof First, note that $0<R_{1} \leq d\left(\alpha_{1}, \partial K\right) \leq\left\|\alpha_{1}\right\|$. Since $B\left[\alpha_{1}, R_{1}\right] \subset K$, it is clear that if $x \in K$ with $\|x\|=R_{1}$, then $x \leq \alpha_{1}$. Since $T$ is non-increasing in $K_{2}$ and $x, \alpha_{1} \in K_{2}$, we have that

$$
T x \geq T \alpha_{1} \geq \alpha_{1} \geq x \quad \text { for all } x \in K \text { with }\|x\|=R_{1} .
$$

If $T x=x$, we have a fixed point such that $\|x\|=R_{1}$, on the contrary, we deduce that $T x \not \leq x$ for all $\|x\|=R_{1}$, which together with (3) implies, by Theorem 1.2, that there exists a nonzero fixed point $x_{1}$ with the desired localization property.

Remark 2.2 Note that if $T \alpha_{1} \neq \alpha_{1}$, in the proof of the previous result, it is showed that the map $T$ has at least one non-zero fixed point $x_{1} \in K$ such that

$$
\min \left\{r_{1}, R_{1}\right\}<\left\|x_{1}\right\|<\max \left\{r_{1}, R_{1}\right\} .
$$

As a direct consequence of Theorem 2.1, we obtain the following 'dual result' for a nonincreasing operator in a solid cone with an upper solution.

Corollary 2.3 Let $N$ be a real Banach space, $K$ be a solid cone and $T: K \rightarrow K$ be a completely continuous operator. Assume that condition (3) is fulfilled together with

(4) there exist $\beta_{1} \in K$, with $T \beta_{1} \leq \beta_{1}$, and $R_{1}>0$ such that $B\left[T \beta_{1}, R_{1}\right] \subset K$;

(5) the map $T$ is monotone non-increasing in the set

$$
K_{5}=\left\{x \in K: R_{1} \leq\|x\| \leq \max \left\{\left\|T \beta_{1}\right\|,\left\|\beta_{1}\right\|\right\}\right\} .
$$

Then the map $T$ has at least one non-zero fixed point $x_{1}$ in $x_{1} \in K \backslash\left\{x \in K ;\|x\|=r_{1}\right\}$, and

$$
\min \left\{r_{1}, R_{1}\right\} \leq\left\|x_{1}\right\| \leq \max \left\{r_{1}, R_{1}\right\}
$$

Proof As in the proof of Theorem 2.1, we deduce that $\left\|T \beta_{1}\right\| \geq R_{1}$.

If $\left\|\beta_{1}\right\|=\left\|T \beta_{1}-\beta_{1}-T \beta_{1}\right\| \leq R_{1}$, then $T \beta_{1}-\beta_{1} \in B\left[T \beta_{1}, R_{1}\right] \subset K$, which implies that $T \beta_{1}-\beta_{1} \geq 0$, i.e., $\beta_{1}$ is a fixed point of $T$. Obviously, if this is the case, $\left\|\beta_{1}\right\|=\left\|T \beta_{1}\right\|=R_{1}$ and the result holds.

On the other hand, if $\left\|\beta_{1}\right\|>R_{1}$, we know that $\beta_{1}, T \beta_{1} \in K_{5}$ and, since $T$ is non-increasing in $K_{5}$ and $T \beta_{1}$ belongs to the interior of $K$, we deduce that $0 \ll T \beta_{1} \leq T\left(T \beta_{1}\right)$, i.e., $T \beta_{1} \in K$ is a non-zero lower solution of the operator $T$.

As consequence, all the conditions of Theorem 2.1 are fulfilled and the result holds.

Now, we give a fixed point result for a non-increasing operator under the assumption of the existence of an upper solution in a cone not necessarily solid, but it verifies an extra condition. 
Theorem 2.4 Let $N$ be a real Banach space, $K$ be a cone that satisfies the following condition:

$$
\text { there exists } \sigma \geq 1 \text { such that for } x, y \in K \text { with }\|x\|=\sigma\|y\| \text { we have } x \geq y \text {, }
$$

and $T: K \rightarrow K$ is a completely continuous operator. Assume that

(6) there exists $\beta_{1} \in K, \beta_{1} \neq 0$, such that $\beta_{1} \geq T \beta_{1}$;

(7) the map $T$ is monotone non-increasing in the set

$$
K_{7}=\left\{x \in K:\left\|\beta_{1}\right\| \leq\|x\| \leq \sigma\left\|\beta_{1}\right\|\right\}
$$

(8) there exists $r_{1}>0$, with $r_{1} \neq R_{1}:=\sigma\left\|\beta_{1}\right\|$, such that $T x \not \leq x$ for all $x \in K$ with $\|x\|=r_{1}$. Then the map $T$ has at least one non-zero fixed point $x_{1} \in K \backslash\left\{x \in K ;\|x\|=r_{1}\right\}$ such that

$$
\min \left\{r_{1}, R_{1}\right\} \leq\left\|x_{1}\right\| \leq \max \left\{r_{1}, R_{1}\right\}
$$

Proof By the property (2.1), we have that if $x \in K$ with $\|x\|=R_{1}$, then $x \geq \beta_{1}$. The definition of $R_{1}$ says that $x, \beta_{1} \in K_{7}$; so, since $T$ is non-increasing on $K_{7}$, we have

$$
T x \leq T \beta_{1} \leq \beta_{1} \leq x \quad \text { for all } x \in K \text { with }\|x\|=R_{1} .
$$

If $T x=x$, we have a fixed point such that $\|x\|=R_{1}$; on the contrary, $T x \nsupseteq x$ for all $x \in K$ such that $\|x\|=R_{1}$ which, together with (8), implies by Theorem 1.2 the existence of a fixed point with the desired localization property.

Remark 2.5 (i) Note that if $T \beta_{1} \neq \beta_{1}$, in the proof of the two previous results, it is showed that the map $T$ has at least one non-zero fixed point $x_{1} \in K$ such that

$$
\min \left\{r_{1}, R_{1}\right\}<\left\|x_{1}\right\|<\max \left\{r_{1}, R_{1}\right\} .
$$

The same conclusion holds if in condition (2.1) we assume that the constant $\sigma>1$. To verify this, it is enough to take into account that if there is a fixed point $x \in K$ with $\|x\|=R_{1}$, then $T x \leq T \beta_{1} \leq \beta_{1} \leq x$ and, as a consequence, $\sigma\left\|\beta_{1}\right\|=\|x\|=\left\|\beta_{1}\right\|$.

(ii) An example of a cone satisfying condition (2.1) is, for instance, the one used in [7]

$$
K=\left\{f \in C[0,1]: f(t)=c t^{2}, c \geq 0\right\} .
$$

Lemma 2.6 Condition (2.1) is equivalent to the following one:

$$
\text { there exists } \sigma \geq 1 \text { such that for } x, y \in K \text { with }\|x\| \geq \sigma\|y\| \text { we have } x \geq y \text {. }
$$

Proof Obviously, if condition (2.2) is fulfilled, then (2.1) also holds.

Suppose now that (2.1) is satisfied, and let $x, y \in K$ be such that $\|x\| \geq \sigma\|y\|$; in consequence, there is $\lambda \geq 1$ such that $\|x / \lambda\|=\sigma\|y\|$. Condition (2.1) shows that $x \geq \lambda y \geq y$, i.e., condition (2.2) holds. This proves the result.

In an analogous way to Corollary 2.3, we arrive at the following 'dual result'.

Corollary 2.7 Let $N$ be a real Banach space, $K$ be a cone that satisfies the condition (2.1) and $T: K \rightarrow K$ be a completely continuous operator. Assume that 
(9) there exists $\alpha_{1} \in K, \alpha_{1} \neq 0$ such that $\alpha_{1} \leq T \alpha_{1}$;

(10) the map $T$ is monotone non-increasing in the set

$$
K_{10}=\left\{x \in K: \min \left\{\left\|\alpha_{1}\right\|,\left\|T \alpha_{1}\right\|\right\} \leq\|x\| \leq \sigma\left\|T \alpha_{1}\right\|\right\} ;
$$

(11) there exists $r_{1}>0$, with $r_{1} \neq R_{1}:=\sigma\left\|T \alpha_{1}\right\|$, such that $T x \not \leq x$ for all $x \in K$ with $\|x\|=r_{1}$.

Then the map $T$ has at least one non-zero fixed point $x_{1} \in K \backslash\left\{x \in K ;\|x\|=r_{1}\right\}$ such that

$$
\min \left\{r_{1}, R_{1}\right\} \leq\left\|x_{1}\right\| \leq \max \left\{r_{1}, R_{1}\right\}
$$

Proof By the property (2.1), we have that if $x \in K$ with $\|x\|=R_{1}$, then $x \geq T \alpha_{1}$.

Suppose now that $\left\|\alpha_{1}\right\| \geq \sigma\left\|T \alpha_{1}\right\|$. From Lemma 2.6, we have that $\alpha_{1} \geq T \alpha_{1}$, which implies that $\alpha_{1}$ is a fixed point with $\left\|\alpha_{1}\right\|=R_{1}$ and $\sigma=1$, so the result holds.

When $\left\|\alpha_{1}\right\|<\sigma\left\|T \alpha_{1}\right\|$, by the definition of $R_{1}$, it is obvious that $\alpha_{1}, T \alpha_{1} \in K_{10}$; so, since $T$ is non-increasing on $K_{10}$, we have

$$
T\left(T \alpha_{1}\right) \geq T\left(\alpha_{1}\right)>0,
$$

and the results holds from Theorem 2.4.

Remark 2.8 Note that if $T \alpha_{1} \neq \alpha_{1}$ or $\sigma>1$, in the proof of the previous result, it is showed that the map $T$ has at least one non-zero fixed point $x_{1} \in K$ such that

$$
\min \left\{r_{1}, R_{1}\right\}<\left\|x_{1}\right\|<\max \left\{r_{1}, R_{1}\right\} .
$$

\section{Non-decreasing operators}

For non-decreasing operators, in the case of an upper solution, it was proved in [4] the following result which is an improvement of those in $[2,3]$.

Theorem 3.1 [4] Let $N$ be a real Banach space, $K$ be a normal solid cone with a normal constant $c \geq 1$ and $T: K \rightarrow K$ be a completely continuous operator. Assume that

(12) there exist $\beta_{1} \in K$, with $T \beta_{1} \leq \beta_{1}$, and $R_{1}>0$ such that $B\left[\beta_{1}, R_{1}\right] \subset K$;

(13) the map $T$ is monotone non-decreasing in the set

$$
K_{13}=\left\{x \in K: \frac{R_{1}}{c} \leq\|x\| \leq c\left\|\beta_{1}\right\|\right\}
$$

(14) there exists $r_{1}>0$, with $r_{1} \neq R_{1}$, such that $T x \not \leq x$ for all $x \in K$ with $\|x\|=r_{1}$.

Then the map $T$ has at least one non-zero fixed point $x_{1}$ in $K$ that either belongs to $K_{13}$ or is such that

$$
\min \left\{r_{1}, R_{1}\right\}<\left\|x_{1}\right\|<\max \left\{r_{1}, R_{1}\right\} .
$$

In the sequel, we prove a fixed point result in this direction for a not necessarily normal cone that satisfies (2.1).

Theorem 3.2 Let $N$ be a real Banach space, $K$ be a solid cone that satisfies condition (2.1), and $T: K \rightarrow K$ be a completely continuous operator. Assume that conditions (12) and (14) hold and 
$\left(13^{\prime}\right)$ the map $T$ is monotone non-decreasing in the set

$$
K_{13^{\prime}}=\left\{x \in K: R_{1} \leq\|x\| \leq \sigma\left\|\beta_{1}\right\|\right\}
$$

Then the map $T$ has at least one non-zero fixed point $x_{1}$ in $K$ that either belongs to $K_{13^{\prime}}$ or is such that

$$
\min \left\{r_{1}, R_{1}\right\}<\left\|x_{1}\right\|<\max \left\{r_{1}, R_{1}\right\}
$$

Proof Since $B\left[\beta_{1}, R_{1}\right] \subset K$, it is clear that if $x \in K$ with $\|x\|=R_{1}$, then $x \leq \beta_{1}$.

Suppose first that there is $\alpha_{1} \in K$ with $\left\|\alpha_{1}\right\|=R_{1}$ and $T \alpha_{1} \geq \alpha_{1}$. Notice that in this case $\alpha_{1} \leq T \alpha_{1} \leq T \beta_{1} \leq \beta_{1}$.

If $\left\|T \alpha_{1}\right\|=\left\|\alpha_{1}-T \alpha_{1}-\alpha_{1}\right\| \leq R_{1}$, then $\alpha_{1}-T \alpha_{1} \in B\left[T \alpha_{1}, R_{1}\right] \subset K$, which implies that $\alpha_{1} \geq T \alpha_{1}$, i.e., $\alpha_{1} \in K_{13^{\prime}}$ is a fixed point of $T$ and the result is fulfilled.

Suppose now that $\left\|T \alpha_{1}\right\|>R_{1}$. If $\left\|T \alpha_{1}\right\| \geq \sigma\left\|\beta_{1}\right\|$ we know, by condition (2.1), that $T \alpha_{1} \geq$ $\beta_{1}$ and, as a consequence, $\beta_{1} \in K_{13^{\prime}}\left(\left\|\beta_{1}\right\| \geq R_{1}\right)$ is a fixed point of $T$.

So, if $R_{1}<\left\|T \alpha_{1}\right\|<\sigma\left\|\beta_{1}\right\|$ we have that $T \alpha_{1} \leq T^{2} \alpha_{1}$ and, arguing as before, we have that either $\alpha_{1}$ or $\beta_{1}$ are fixed points of $T$, or $R_{1}<\left\|T^{2} \alpha_{1}\right\|<\sigma\left\|\beta_{1}\right\|$.

By recurrence we verify that the set $P=\left\{T^{n} \alpha_{1}\right\}_{n \in \mathbb{N}} \subset K_{13^{\prime}}$.

Since $T$ is monotone non-decreasing on $K_{13^{\prime}}$, we have that the same holds in $P$. Moreover, $T(P) \subset\left[\alpha_{1}, \beta_{1}\right]$. Now, the completely continuous character of the operator $T$ implies that $\overline{T(P)}$ is a compact set of $N$. We can ensure the existence of a fixed point on $P \subset K_{13^{\prime}}$ from [8, Proposition 1.1.7].

Now suppose that $T x \ngtr x$ for all $x \in K$ with $\|x\|=R_{1}$. By (14) there exists $r_{1}>0$ such that $T x \not \leq x$ for all $x \in K$ with $\|x\|=r_{1}$. Therefore by Theorem 1.2 there exists a non-zero fixed point.

Remark 3.3 An example of a solid cone satisfying condition (2.1) is the following one:

$$
K=\left\{c f(t): f \in L^{\infty}([0,1]), f \geq 0 \text { a.e. } t \in[0,1],\|f\|_{1}>0, c \geq 0\right\}
$$

Now, we give a result under the assumption of the existence of a lower solution.

Theorem 3.4 Let $N$ be a real Banach space, $K$ be a normal cone (not necessarily solid) with a normal constant $c \geq 1$ that satisfies condition (2.1), and $T: K \rightarrow K$ be a completely continuous operator. Assume that there is a lower solution as in (9), and

(15) the map $T$ is monotone non-decreasing in the set

$$
K_{15}=\left\{x \in K: \frac{\left\|\alpha_{1}\right\|}{c} \leq\|x\| \leq c \sigma\left\|\alpha_{1}\right\|\right\}
$$

(16) there exists $r_{1}>0$, with $r_{1} \neq R_{1}:=\sigma\left\|\alpha_{1}\right\|$, such that $T x \ngtr x$ for all $x \in K$ with $\|x\|=r_{1}$. Then the map $T$ has at least one non-zero fixed point $x_{1}$ in $K$ that either belongs to $K_{15}$ or is such that

$$
\min \left\{r_{1}, R_{1}\right\}<\left\|x_{1}\right\|<\max \left\{r_{1}, R_{1}\right\}
$$


Proof By the property (2.1), we have that if $x \in K$ with $\|x\|=R_{1}$, then $x \geq \alpha_{1}$.

Suppose first that we can choose $\beta_{1} \in K$ with $\left\|\beta_{1}\right\|=R_{1}$ and $T \beta_{1} \leq \beta_{1}$. Since $\alpha_{1} \leq \beta_{1}$ and due to the normality of the cone $K$, we have that $\left[\alpha_{1}, \beta_{1}\right] \subset K_{15}$, which implies that $T$ is nondecreasing on $\left[\alpha_{1}, \beta_{1}\right]$. Then we can apply Theorem 1.1 to ensure the existence of the extremal fixed points of $T$ on $\left[\alpha_{1}, \beta_{1}\right]$.

Now suppose that $T x \not \leq x$ for all $x \in K$ with $\|x\|=R_{1}$. By (16) there exists $r_{1}>0$ such that $T x \nsupseteq x$ for all $x \in K$ with $\|x\|=r_{1}$. Therefore by Theorem 1.2, there exists a non-zero fixed point $x_{1}$ in the required set.

Remark 3.5 We stress that the above theorems can be combined to prove the existence of multiple fixed points. The idea is to use a nesting argument similar to those utilized, for example, in $[9,10]$, where the authors used the classical fixed point index, and in [11, 12], where Theorem 1.2 was used. In the next section we do this in the case of the existence of two non-trivial fixed points, and we refer to Theorem 3.4 of [4] to give an idea of the type of results that may be stated in the case of $n$ fixed points.

\section{Applications to a nonlocal BVP}

We now discuss the existence of positive solutions of the nonlocal boundary value problem (BVP)

$$
\begin{aligned}
& u^{(4)}(t)=\lambda g(t) f(u(t)), \quad t \in(0,1), \\
& u^{\prime}(0)=0, \quad \theta_{1} u^{\prime}(1)+u\left(\xi_{1}\right)=0, \\
& u^{\prime \prime \prime}(0)=0, \quad \theta_{2} u^{\prime \prime \prime}(1)+u^{\prime \prime}\left(\xi_{2}\right)=0,
\end{aligned}
$$

where, for $i=1,2, \theta_{i} \in \mathbb{R}$ and $\xi_{i} \in[0,1], g \in L^{1}[0,1], g \geq 0$ a.e. and $f:[0, \infty) \rightarrow[0, \infty)$ is continuous. BVP (4.1)-(4.3) that has been studied in [13] models the displacement of a beam with feedback controllers; in particular, the boundary conditions mean that the shear force and the angular attitude vanish at $t=0$, and in $t=1$ they are related to the displacement and to the bending moment registered in other points of the beam.

This BVP can be rewritten as a Hammerstein integral equation of the form

$$
u(t)=\int_{0}^{1} k(t, s) \lambda g(s) f(u(s)) d s:=T u(t)
$$

where the Green's function $k(t, s)$ and its properties are given in the following result.

Lemma 4.1 [13] Let $\theta_{1}+\xi_{1}>1$ and $\theta_{2}+\xi_{2}>1$. The Green's function $k(t, s)$ for the linear fourth-order boundary value problem

$$
\begin{array}{ll}
u^{(4)}(t)=y(t), & t \in(0,1), \\
u^{\prime}(0)=0, & u^{\prime \prime \prime}(0)=0, \quad \theta_{1} u^{\prime}(1)+u\left(\xi_{1}\right)=0, \quad \theta_{2} u^{\prime \prime \prime}(1)+u^{\prime \prime}\left(\xi_{2}\right)=0,
\end{array}
$$


is given by

$$
\begin{aligned}
k(t, s)= & \theta_{2}\left(\theta_{1}+\frac{\xi_{1}^{2}}{2}-\frac{t^{2}}{2}\right)-\frac{\theta_{1}}{2}(1-s)^{2}+\left\{\begin{array}{l}
\left(\theta_{1}+\frac{\xi_{1}^{2}}{2}-\frac{t^{2}}{2}\right)\left(\xi_{2}-s\right), \quad s \leq \xi_{2}, \\
0, \quad s>\xi_{2},
\end{array}\right. \\
& -\left\{\begin{array}{ll}
\frac{1}{6}\left(\xi_{1}-s\right)^{3}, \\
0, \quad s>\xi_{1},
\end{array} \quad s \leq \xi_{1}, \quad+ \begin{cases}\frac{1}{6}(t-s)^{3}, & s \leq t, \\
0, & s>t .\end{cases} \right.
\end{aligned}
$$

Moreover, for $(t, s) \in[0,1] \times[0,1]$, we have

$$
\hat{c} k(0, s) \leq k(t, s) \leq k(0, s):=\Phi(s),
$$

where

$$
\hat{c}:=1-\frac{1}{2 \theta_{1}+\xi_{1}^{2}} .
$$

Note that $\theta_{1}+\xi_{1}>1$ implies that $2 \theta_{1}+\xi_{1}^{2}>1$ and so $0<\hat{c}<1$. Now, with the above conditions, it is routine to prove that $T: C[0,1] \rightarrow C[0,1]$ leaves invariant the cone

$$
K=\left\{u \in C[0,1]: \min _{t \in[0,1]} u(t) \geq \hat{c}\|u\|\right\}
$$

where in $C[0,1]$ we are considering the supremum norm $\|u\|=\sup \{u(t): t \in[0,1]\}$. It is also known that $K$ is a normal solid cone with a normal constant $c=1$.

We will make use of the numbers

$$
\gamma_{*}=\inf _{t \in[0,1]} \int_{0}^{1} k(t, s) g(s) d s, \quad \gamma^{*}=\sup _{t \in[0,1]} \int_{0}^{1} k(t, s) g(s) d s,
$$

note that $\gamma_{*}=1 / M$ and $\gamma^{*}=1 / m$, in the notation of [13].

Now we present the main result of this section.

Theorem 4.2 Assume that the hypotheses in Lemma 4.1 hold. Moreover, let $\beta_{1}, \alpha_{2}, R_{1}, R_{2} \in$ $(0,+\infty)$ be such that

$$
\beta_{1}<\hat{c} R_{2}, \quad \alpha_{2} \geq R_{2}\left(2\left(2 \theta_{1}+\xi_{1}^{2}\right)-1\right) \quad \text { and } \quad \beta_{1} \geq R_{1}\left(2\left(2 \theta_{1}+\xi_{1}^{2}\right)-1\right) .
$$

Assume that $g$ satisfies that $\gamma_{*}>0$ and, moreover,

(i) $f$ is non-decreasing on $\left[\hat{c} R_{1}, \beta_{1}\right]$;

(ii) $f$ is non-increasing on $\left[\hat{c} R_{2}, \alpha_{2}\right]$;

(iii) $\lim _{u \rightarrow 0^{+}} \frac{f(u)}{u}=+\infty$ and $\lim _{u \rightarrow+\infty} \frac{f(u)}{u}=0$.

Then BVP (4.1)-(4.3) has at least two positive solutions for any $\lambda>0$ satisfying

$$
\frac{\alpha_{2}}{\gamma_{*} f\left(\alpha_{2}\right)} \leq \lambda \leq \frac{\beta_{1}}{\gamma^{*} f\left(\beta_{1}\right)}
$$

Proof The main idea in the proof is to apply Theorems 2.1 and 3.1 in two disjoint conical shells in order to get two different non-trivial fixed points. Firstly, we are going to check that the conditions of Theorem 2.1 are satisfied. 
(1.a) $\alpha_{2} \leq T \alpha_{2}$ and there exists $R_{2}>0$ such that $B\left[\alpha_{2}, R_{2}\right] \subset K$.

We have $\alpha_{2} \leq T \alpha_{2}$ because of the inequality $\frac{\alpha_{2}}{\gamma_{*} f\left(\alpha_{2}\right)} \leq \lambda$. On the other hand, the inequality $\alpha_{2} \geq R_{2}\left(2\left(2 \theta_{1}+\xi_{1}^{2}\right)-1\right)$ implies that $B\left[\alpha_{2}, R_{2}\right] \subset K$. Indeed, let $u \in B\left[\alpha_{2}, R_{2}\right]$, that is,

$$
\alpha_{2}-R_{2} \leq u(t) \leq \alpha_{2}+R_{2} \quad \text { for all } t \in[0,1]
$$

Since $2 \theta_{1}+\xi_{1}^{2}>1$, we have $\alpha_{2}>R_{2}$ and then $u(t)>0$ for all $t \in[0,1]$. Moreover, it is easy to check that

$$
\min _{t \in[0,1]} u(t) \geq \alpha_{2}-R_{2} \geq \hat{c}\left(\alpha_{2}+R_{2}\right) \geq \hat{c}\|u\|,
$$

which means that $u \in K$.

(1.b) The map $T$ is monotone non-increasing in the set

$$
K_{2}=\left\{x \in K: R_{2} \leq\|x\| \leq\left\|\alpha_{2}\right\|\right\}
$$

This fact is a consequence of the assumption (ii).

(1.c) There exists $r_{2}>0$, with $r_{2} \neq R_{2}$, such that $T x \nsupseteq x$ for all $x \in K$ with $\|x\|=r_{2}$.

By the second part of the assumption (iii), we have this result for $r_{2}>R_{2}$ large enough.

So, Theorem 2.1 implies the existence of a solution $x_{2} \in K$ such that

$$
R_{2} \leq\left\|x_{2}\right\|<r_{2}
$$

Now, we are going to check that the conditions of Theorem 3.1 are satisfied.

(2.a) $T \beta_{1} \leq \beta_{1}$ and there exists $R_{1}>0$ such that $B\left[\beta_{1}, R_{1}\right] \subset K$.

We have $T \beta_{1} \leq \beta_{1}$ because of the inequality $\lambda \leq \frac{\beta_{1}}{\gamma^{*} f\left(\beta_{1}\right)}$. Again,

$\beta_{1} \geq R_{1}\left(2\left(2 \theta_{1}+\xi_{1}^{2}\right)-1\right)$ implies that $B\left[\beta_{1}, R_{1}\right] \subset K$ by reasoning as in the proof of claim (1.a).

(2.b) The map $T$ is monotone non-decreasing in the set

$$
K_{1}=\left\{x \in K: R_{1} \leq\|x\| \leq\left\|\beta_{1}\right\|=\beta_{1}\right\} .
$$

This fact is a consequence of the assumption (i).

(2.c) There exists $r_{1}>0$, with $r_{1} \neq R_{1}$, such that $T x \not \leq x$ for all $x \in K$ with $\|x\|=r_{1}$.

By the first part of the assumption (iii), we have this result for $0<r_{1}<R_{1}$ small enough.

Then, applying Theorem 3.1, we get the existence of a solution $x_{1} \in K$ such that $r_{1}<$ $\left\|x_{1}\right\| \leq \beta_{1}$. Since $\beta_{1}<\hat{c} R_{2}$, we have that $x_{1} \neq x_{2}$ and the theorem is proven.

The following example illustrates our previous theorem.

Example 4.3 We consider the BVP

$$
u^{(4)}(t)=\lambda f(u(t)), \quad t \in(0,1)
$$




$$
u^{\prime}(0)=u^{\prime \prime \prime}(0)=\frac{5}{6} u^{\prime}(1)+u\left(\frac{1}{5}\right)=\frac{2}{7} u^{\prime \prime \prime}(1)+u^{\prime \prime}\left(\frac{4}{5}\right)=0
$$

with

$$
f(u)=\sqrt{u}+\frac{300 e^{-e^{20(2-u)}}\left(\tan ^{-1}(21-u)+\frac{\pi}{2}\right)}{\pi} .
$$

In Figures 1, 2 and 3 you can see the behavior of the function $f$ on different intervals. In this example $g(t)=1$, and by [13] we know that $\gamma_{*}=\frac{3,677}{31,500}$ and $\gamma^{*}=\frac{23,809}{63,000}$. Moreover, since $\theta_{1}=\frac{5}{6}$ and $\xi_{1}=\frac{1}{5}$, we have $\hat{c}=\frac{53}{128}$.

Now, it is easy to check that all the assumptions of Theorem 4.2 are satisfied by taking $\beta_{1}=\frac{3}{2}, \alpha_{2}=20, R_{1}=\frac{1}{2}$ and $R_{2}=8$. So, by Theorem 4.2, BVP (4.8)-(4.9) has at least two positive solutions provided that

$0.74665 \leq \lambda \leq 3.24075$

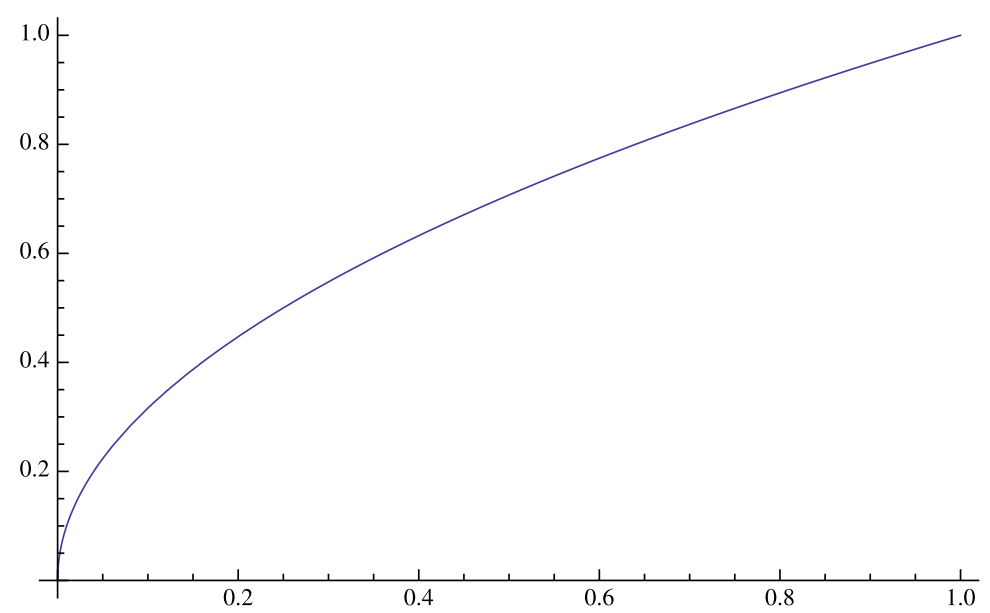

Figure 1 Graph of $f$ on $[0,1]$.

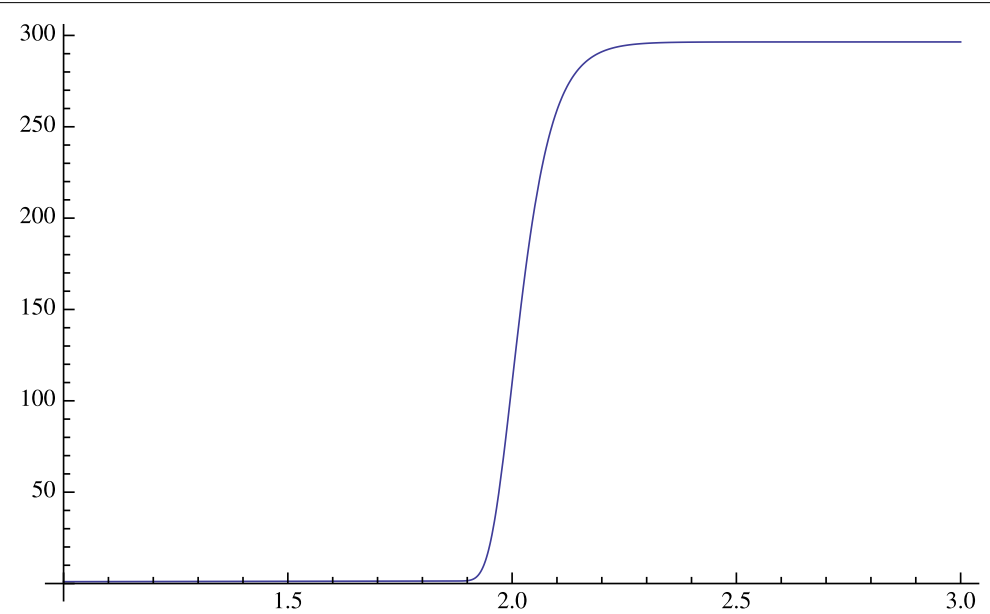

Figure 2 Graph of $f$ on $[1,3]$. 


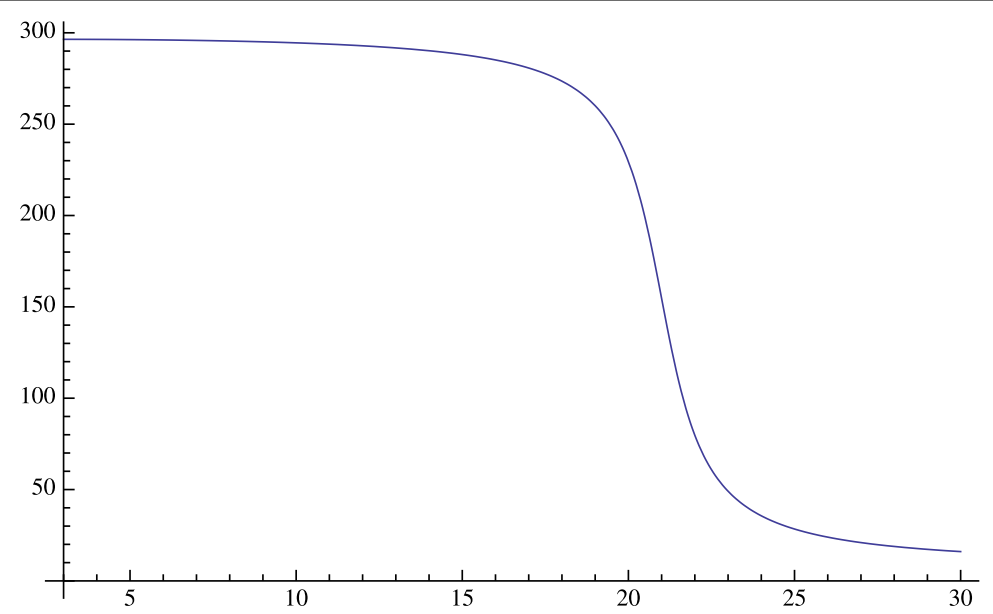

Figure 3 Graph of $f$ on $[3,30]$.

\section{Competing interests}

The authors declare that they have no competing interests.

\section{Authors' contributions}

All the authors contributed equally and significantly in writing this article. All the authors read and approved the final manuscript.

\section{Author details}

${ }^{1}$ Departamento de Análise Matemática, Facultade de Matemáticas, Universidade de Santiago de Compostela, Campus Vida, Santiago, 15782, Spain. ${ }^{2}$ Departamento de Matemáticas, Universidade de Vigo, Pabellón 3 (Edifico Físicas), Campus de Ourense, Ourense, 32004, Spain. ${ }^{3}$ Dipartimento di Matematica ed Informatica, Università della Calabria, Arcavacata di Rende, Cosenza, 87036, Italy.

\section{Acknowledgements}

Alberto Cabada and José Ángel Cid were partially supported by Ministerio de Educación y Ciencia, Spain, and FEDER Project MTM2010-15314. This paper was partially written during the visit of Gennaro Infante to the Departamento de Análise Matemática of the Universidade de Santiago de Compostela. Gennaro Infante is grateful to the people of the aforementioned Departamento for their kind and warm hospitality.

\section{Received: 6 March 2013 Accepted: 26 April 2013 Published: 13 May 2013}

\section{References}

1. Persson, H: A fixed point theorem for monotone functions. Appl. Math. Lett. 19, 1207-1209 (2006)

2. Cabada, A, Cid, JA: Existence of a non-zero fixed point for nondecreasing operators via Krasnoselskii's fixed point theorem. Nonlinear Anal. 71, 2114-2118 (2009)

3. Cid, JA, Franco, D, Minhós, F: Positive fixed points and fourth-order equations. Bull. Lond. Math. Soc. 41, 72-78 (2009)

4. Franco, D, Infante, G, Perán, J: A new criterion for the existence of multiple solutions in cones. Proc. R. Soc. Edinb. A 142, 1043-1050 (2012)

5. Zeidler, E: Nonlinear Functional Analysis and Its Applications. I. Fixed-Point Theorems. Springer, New York (1986)

6. Amann, $\mathrm{H}$ : On the number of solutions of nonlinear equations in ordered Banach spaces. J. Funct. Anal. 11, 346-384 (1972)

7. Cabada, A, Cid, JA: A note on fixed point theorems for T-monotone operators. Comput. Math. Appl. 47, 853-857 (2004)

8. Heikkilä, S, Lakshmikantham, V: Monotone Iterative Techniques for Discontinuous Nonlinear Differential Equations. Monographs and Textbooks in Pure and Applied Mathematics, vol. 181. Dekker, New York (1994)

9. Infante, G, Webb, JRL: Nonlinear nonlocal boundary value problems and perturbed Hammerstein integral equations. Proc. Edinb. Math. Soc. 49, 637-656 (2006)

10. Lan, KQ: Multiple positive solutions of semilinear differential equations with singularities. J. Lond. Math. Soc. 63 , 690-704 (2001)

11. Graef, JR, Qian, C, Yang, B: Multiple symmetric positive solutions of a class of boundary value problems for higher order ordinary differential equations. Proc. Am. Math. Soc. 131, 577-585 (2003)

12. Karakostas, GL, Tsamatos, PCh: Existence of multiple positive solutions for a nonlocal boundary value problem. Topol. Methods Nonlinear Anal. 19, 109-121 (2002)

13. Infante, G, Pietramala, P: The displacement of a sliding bar subject to nonlinear controllers. In: Proceedings of the International Conference on Differential \& Difference Equations and Applications. Springer, Berlin (in press) 
doi:10.1186/1687-1812-2013-125

Cite this article as: Cabada et al.: New criteria for the existence of non-trivial fixed points in cones. Fixed Point Theory and Applications 2013 2013:125.

Submit your manuscript to a SpringerOpen ${ }^{\circ}$ journal and benefit from:

- Convenient online submission

- Rigorous peer review

- Immediate publication on acceptance

- Open access: articles freely available online

- High visibility within the field

- Retaining the copyright to your article

Submit your next manuscript at $\gg$ springeropen.com 